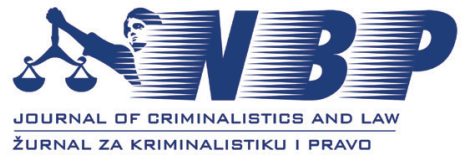

NBP 2021, Vol. 26, Issue 1, pp. 61-72

doi:10.5937/nabepo26-32292

Original scientific paper

\title{
Effect of Grip Size and Grip Strength on Pistol Marksmanship in Police Officers: A Pilot Study
}

\author{
${ }^{1}$ Robin M. Orr ${ }^{1},{ }^{2}$ Anthony Rofe, ${ }^{3}$ Ben Hinton, ${ }^{4}$ Jay Dawes, ${ }^{5}$ Gianpiero Greco, \\ ${ }^{6}$ Robert Lockie \\ ${ }^{1}$ Bond University, Tactical Research Unit, Robina, Qld, Australia \\ ${ }^{2}$ Bond University, Faculty of Health Sciences and Medicine, Robina, Qld, Australia \\ ${ }^{3}$ New South Wales Police Force, Australia \\ ${ }^{4}$ Oklahoma State University, Tactical Fitness and Nutrition Lab, CA, USA \\ ${ }^{5}$ Ministry of Interior, Public Security Department, State Police, Milan, Italy \\ ${ }^{6}$ California State University, Department of Kinesiology, Fullerton, CA, USA
}

Submitted: 2021-05-18 • Accepted: 2021-06-07 • Published: 2021-06-14

\begin{abstract}
Police officers may be required to use their firearms in self-defence. The purpose of this study was to evaluate the relationships between hand grip size and strength with pistol shooting accuracy in police officers. Twelve (age $=38.08 \pm 6.24$ years; height $=174.42 \pm 7.33 \mathrm{~cm}$ ) police officers had their hand sizes (palm width and hand span) and hand grip strength measured. Handgrip dynamometer was set at a Glock 17 pistol's grip width $(50 \mathrm{~mm})$. The officers fired 10 rounds from their service pistols at a stationary target. Independent samples t-tests were performed to identify differences between the sexes. Correlations were used to investigate relationships between measures of hand size, strength, and marksmanship. Alpha levels were set at $\mathrm{p}<0.05$. Male officers were significantly stronger $(p=0.01)$ and had a bigger hand width $(p=0.03)$, but not hand span. There were no significant differences in marksmanship between the sexes. Neither hand size nor grip strength had a significant impact on marksmanship even though there were strong and significant relationships between hand size (span and MCP) and grip strength. A V-shaped curve appears to exist between grip strength and marksmanship and hand span and marksmanship, with a potential influencing factor being the standard sizing of the pistol grip. Keywords: law enforcement, shooting, pistol accuracy, firearm.
\end{abstract}

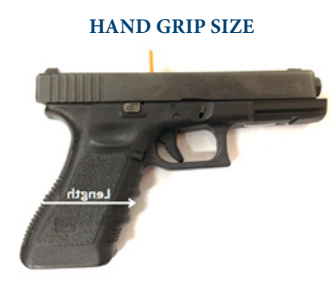

HAND GRIP STRENGTH
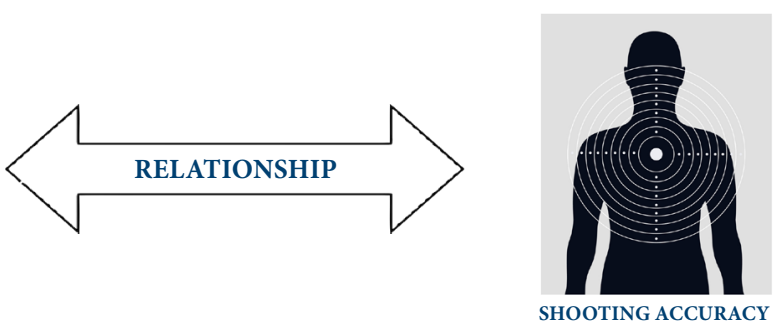

1 Corresponding author: rorr@bond.edu.au

Citation: Orr, R. M., Rofe, A., Hinton, B., Dawes, J., Greco, G., \& Lockie, R. (2021). Effect of grip size and grip strength on pistol marksmanship in police officers: A pilot study. NBP. Nauka, bezbednost, policija, 26(1), 61-72. doi:10.5937/nabepo26-32292 


\section{INTRODUCTION}

Proficiency in pistol marksmanship can mean the difference between life and death for police officers, their partners, and innocent bystanders. Indeed, $70-80 \%$ of police officers who discharge their firearm do so for self or partner protection, and approximately 20-30\% discharge their firearm for bystander safety (Geller \& Scott, 1992). However, research suggests that up to $65 \%$ of police shots miss their target when engaging an armed suspect (Binder \& Fridell, 1984; Charles \& Copay, 2003; Matulia, 1985). This accuracy rate is concerning, as this fact places police officers at a greater risk of being wounded or killed. Furthermore, poor accuracy may also result in innocent civilians being struck by a missed shot.

Grip strength is thought to play an important role in police officer marksmanship (Muirhead et al., 2019; Orr et al., 2017). In a study of 169 police recruits, Orr et al. (2017) found that recruits who passed their initial marksmanship shoot had significantly $(\mathrm{p}<.001)$ greater left and right hand grip strength scores than those who failed. Furthermore, the significant correlations $(\mathrm{p}<.0001)$ between grip strength and marksmanship performance were better in the non-dominant hand (left hand: $r=.475$ ) than the dominant hand (right hand: $r=.398$ ). Conversely, Muirhead et al. (2019) found that while grip strength was significantly correlated to marksmanship scores in a dynamic shooting scenario $(\mathrm{r}=-.367, \mathrm{p}<.05)$, it did not correlate to the other marksmanship shooting events (static shoot, $\mathrm{r}=-.001$; positive identification shoot, $r=0.040$ ). Muirhead et al. (2019) detailed a potential explanation for the conflicting results between studies in that there were no correlations between the different shooting scenarios. In addition to static and dynamic shooting scenarios, research has shown that increases in distance can negatively impact accuracy and variance (Dopsaj et al., 2020; Dopsaj et al., 2019). As such, an officer may have high marksmanship scores on one shooting engagement, but not on a different engagement.

The manner in which grip strength is measured is also of importance. Liao (2016) in a study of 72 healthy male and female adults investigated the relationships between hand sizes (categorised as small, medium and large) and grip strengths with the dynamometer at different widths $(47.6,60.3$, and $73.0 \mathrm{~mm})$. It was discovered that hand length did influence the participants ability to exert maximal force, suggesting that dynamometers should be adjusted based on hand size when measuring hand grip strength. As such, it is unsurprising that the typical protocol for measuring hand grip strength requires the dynamometer to be adjusted to accommodate hand size (Dawes et al., 2017).

Hand grip size, or hand span, is significantly correlated with grip strength whereby individuals with smaller hands, like those more likely to be found in female officers, may not able to exert as much force as those with larger hands (Orr et al., 2017). However, while Anderson and Plecas (2000) found that $2^{\text {nd }}$ ray length was strongly correlated to grip strength $(\mathrm{r}=0.58, \mathrm{p}<.05)$ and shooting scores $(\mathrm{r}=.28, \mathrm{p}<.05)$, no other hand width or length measures were significant. A greater understanding of how hand size impacts grip strength, as well as marksmanship, warrants further consideration. 
Unfortunately, the width of the officer's pistol is pre-determined and is not, as yet, adjusted to the specific hand size of each individual. Noting that several of the aforementioned studies (Anderson \& Plecas, 2000; Charles \& Copay, 2001; Charles \& Copay, 2003) did not provide detail as to the setting of the dynamometer for measuring grip strength, those that did (Orr et al. (2017), following the protocol of Dortkamph (1987) used the protocol of adjusting the dynamometers' grip width to suit the individual. As such the question arises as to whether hand grip strength, using a dynamometer set to the width of the pistol stock, would be related to marksmanship scores. The purpose of this study was to evaluate the relationships between grip size and grip strength, with pistol shooting accuracy (marksmanship) in fully qualified police officers, with a dynamometer set to the width of the pistol stock.

\section{METHODS}

\section{Experimental approach to the problem}

Qualified police officers had their grip size and, using a dynamometer set to width of a pistol stock for occupational specificity, grip strength measured. Officers then engaged in a marksmanship assessment using 10 rounds and standard police issue pistol, with accuracy measured as distance from centre of the target.

\section{Participants}

Qualified police officers $(\mathrm{n}=12$; mean age $=38.08 \pm 6.24$ years; mean height $=$ $174.42 \pm 7.33 \mathrm{~cm}$ ) were recruited from an Australian state police force. Of these officers six were male (mean age $=39.33 \pm 6.68$ years; mean height $=178.33 \pm 6.15$ $\mathrm{cm}$ ) and six were female (mean age $=36.83 \pm 6.11$ years; mean height $=170.50 \pm$ $6.60 \mathrm{~cm}$ ). Participants were recruited as a sample of convenience. Data were collected at the Australian state police force's training college, using their standard training range, and was coordinated by an agency Police Liaison Officer. The data was non-identifiable when provided to the investigators for analysis. Ethics approval for this study was provided by Bond University's Human Research Ethics Committee (RO1898) with individuals providing informed consent.

\section{Measurements and procedures}

Age and height measures were self-reported with the latter found to be reliable in police officers (Dawes et al., 2019). Qualified police officers had their hand size measures taken first followed by their grip strength measurement. After these measures were taken, they performed their standard departmental marksmanship assessments. 


\section{Grip size and strength}

The weapon master hand was used for all measures. In this case, for all participants, it was their right hand. The firsthand size measurement was palm width across the metacarpophalangeal joints (MCP). The second measurement was 'hand span' which was measured from the tip of the pollex to the tip of the $5^{\text {th }}$ phalange. This measure was taken with the hand spread as wide as possible. All measures were performed using a physio liberty tape measure and recorded to the nearest $\mathrm{mm}$. Maximal grip strength, again of the master weapon hand, was measured using a Jamar hand grip dynamometer (Sammons Preston, Boilingbrook IL, United States) set at the width of the Glock pistol (50 mm: Figure 1). The Jamar dynamometer has a high intra-rater reliability (ICC 0.94-0.98) (Peolsson et al., 2001) and is a valid measure of grip strength $(r=0.9998)$ (Mathiowetz, 2002). The officer held the dynamometer out in front of their body adopting a firing position. They were instructed to progressively squeeze the hand to obtain a maximum grip draw. The average grip strength score of three attempts were used as the final result.

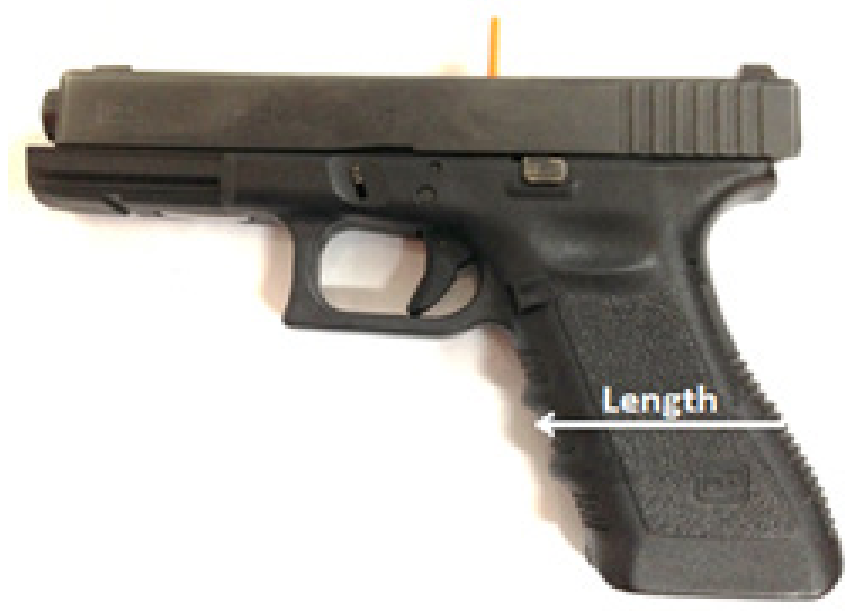

Figure 1. Standard issue $9 \mathrm{~mm}$ Glock pistol used in the study with measurements used for grip strength

\section{Marksmanship measure}

The marksmanship assessment was conducted using Z-4 police targets with a centre aiming point marked by a disc $70 \mathrm{~mm}$ in diameter (See Figure 2). Officers fired 10 rounds at the target centre from a standard issued Glock pistol (Glock 17 standard) using allocated police ammunition from a static, standing, unsupported position seven meters from the target. Marksmanship measures were those previously reported in the literature (Carbone et al., 2014) being, distance from the centre of the target (DCOT), horizontal displacement (X) and vertical displacement (Y). DCOT was measured in $\mathrm{mm}$, from each of the 10 falls of shot to the centre of the target and averaged. The $\mathrm{X}$ displacement was defined as the distance between the two farthest horizontally displaced falls of shot measured in millimetres and Y displacement as 
the distance between the two farthest vertically displaced falls of shot. $\mathrm{X}$ and $\mathrm{Y}$ displacements were used to increase sensitivity of the marksmanship results through isolating potential influencing factors along these two axes.

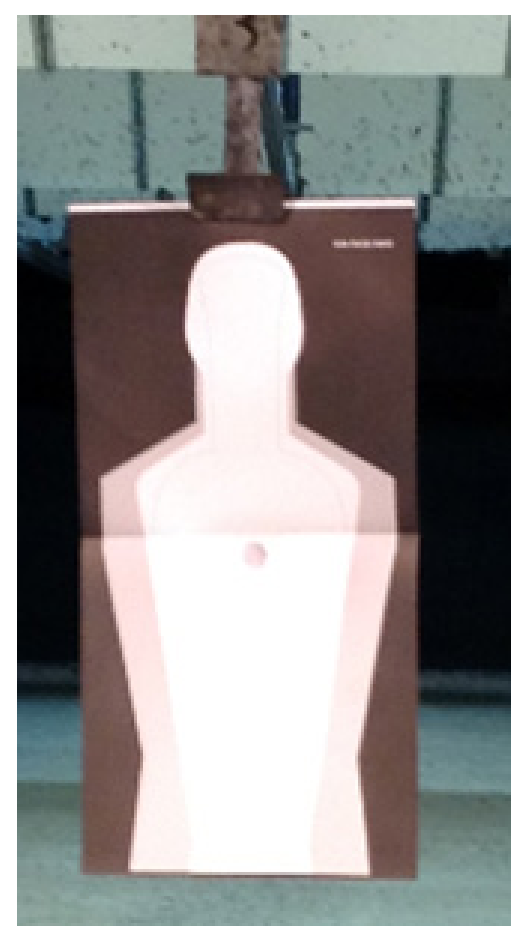

Figure 2. Police marksmanship target

\section{Statistical analyses}

The SPSS Version 26 statistical software package (SPSS Inc., Delaware, USA) was used for descriptive and inferential analysis. Mean and standard deviations were calculated. Following assumption of normality, satisfied using Shapiro-Wilk test, and of homogeneity of variances, via a Levene's test, an independent samples t-test were used to investigate differences between the sexes for all measures. Effect sizes (Cohen's $d$ ) for the between-sex comparisons were also calculated from the difference between the means divided by the pooled standard deviations (Cohen, 1988). A $d$ less than 0.2 was considered a trivial effect; 0.2 to 0.6 a small effect; 0.6 to 1.2 a moderate effect; 1.2 to 2.0 a large effect; 2.0 to 4.0 a very large effect; and 4.0 and above an extremely large effect (Hopkins, 2004). Pearson's correlations, or Spearman's Rho (for non-normally distributed statistics), by total sample and individual sex, were used to determine relationships between grip strength and marksmanship, grip size and marksmanship and grip strength and grip size $(\mathrm{p}<0.05)$. The correlation strength was designated as: an $\mathrm{r}$ between 0 to \pm 0.3 was considered small; \pm 0.31 to \pm 0.49 , moderate; \pm 0.5 to \pm 0.69 , large; \pm 0.7 to \pm 0.89 , very large; and \pm 0.9 to \pm 1 near perfect relationship (Hopkins, 2002). 


\section{RESULTS}

The results were taken from all 12 participants; however, one of the participants did not undergo the hand MCP or grip strength measurements. Their results were still included in the statistical analysis for the other measures. Descriptive values for the hand size, grip strength and marksmanship measures are detailed in Table 1. While height $(\mathrm{p}=0.059)$ and hand span $(\mathrm{p}=0.068)$ approached significance, only MCP $(\mathrm{t}[5.534]=-2.988, \mathrm{p}=0.027$ unequal variances) and grip strength $(\mathrm{t}[9]=-3.086, \mathrm{p}$ $=0.013$ ) were significantly different between the sexes, both with large effect sizes.

Table 1. Descriptive data for the complete sample and for male and female officers separately

\begin{tabular}{lcccccc}
\hline Measure & Total & Male Officers & $\begin{array}{c}\text { Female } \\
\text { Officers }\end{array}$ & $\mathbf{p}$ & $\mathbf{d}$ & $\begin{array}{c}\mathbf{d} \\
\text { strength }\end{array}$ \\
\hline Age (years) & $38.08(6.24)$ & $39.33(6.68)$ & $36.83(6.11)$ & 0.51 & 0.39 & Small \\
Height $(\mathrm{cm})$ & $174.42(7.33)$ & $178.33(6.15)$ & $170.50(6.60)$ & 0.06 & 1.23 & Large \\
Hand span $(\mathrm{mm})$ & $21.13(1.52)$ & $21.92(1.11)$ & $20.33(1.54)$ & 0.07 & 1.18 & Moderate \\
MCP $(\mathrm{mm})^{\wedge}$ & $8.13(0.50)$ & $8.48(0.11)^{*}$ & $7.83(0.54)$ & $0.03^{*}$ & 1.67 & Large \\
Grip strength $(\mathrm{kg})^{\wedge}$ & $41.97(12.33)$ & $51.20(7.54)^{\star}$ & $34.28(10.11)$ & $0.01^{\star}$ & 1.9 & Large \\
DCOT $(\mathrm{mm})$ & $116.70(30.54)$ & $103.00(35.32)$ & $130.40(18.80)$ & 0.12 & -0.97 & Moderate \\
X-Axis $(\mathrm{mm})$ & $205.17(30.54)$ & $195.67(58.00)$ & $214.67(68.59)$ & 0.62 & -0.3 & Small \\
Y-Axis $(\mathrm{mm})$ & 306.08 & 263.00 & $349.17(84.08)$ & 0.20 & -0.79 & $\begin{array}{c}\text { Moder- } \\
\text { ate }\end{array}$ \\
\hline
\end{tabular}

$\wedge \mathrm{n}=11:^{*}$ Significantly different from female officers $\mathrm{p}<.05$

Table 2 shows the results of the Pearson's correlation. When all participants were considered there was a strong positive correlation between hand span and grip strength $(\mathrm{r}=.745)$ and MCP width and grip strength $(\mathrm{r}=.762)$. However, there were no other significant correlations between hand size and hand grip measures or shooting performance. Likewise, there are no significant correlations between measures in the female (Table 3) or the male (Table 4) officers. When viewed graphically (Figure 2), there was a general trend towards lower (better) DCOT scores with higher grip strength for female officers. Conversely, male officers trended towards higher (worse) DCOT scores with increasing grip strength. Of note, five of the six female scores were below the mean for grip strength and above the mean for DCOT. Similarly, Figure 3 details a general trend towards lower (better) DCOT scores with a larger hand span for female officers. In contrast, male officers trended towards higher (worse) DCOT scores as hand span increased. When both Figure 3 and Figure 4 are considered holistically (male and female results), an emerging V-Shaped curve $(<)$ is seen. 
Table 2. Pearson Correlations for total participants $(n=12)$

\begin{tabular}{lcccccc}
\hline & Hand Span & MCP & Grip Strength & DCOT & X-Axis & Y-Axis \\
\hline Hand Span & 1 & & & -0.29 & -0.42 & 0.11 \\
$\mathrm{MCP}^{\wedge}$ & $0.40 \sim$ & 1 & & $-0.36 \sim$ & $-0.08 \sim$ & $-0.35 \sim$ \\
Grip & $.75^{\star *}$ & $.69 \sim^{*}$ & 1 & -0.25 & -0.28 & -0.08 \\
Strength^ & & & & & \\
\hline
\end{tabular}

$\wedge_{\mathrm{n}}=11, \sim$ Spearman's Rho, Correlation is significant at ${ }^{\star} \mathrm{p}<0.5 ;{ }^{\star *} \mathrm{p}<.01$

Table 3. Pearson Correlations for female participants $(n=6)$

\begin{tabular}{lcccccc}
\hline & Hand Span & MCP & Grip Strength & DCOT & X- Axis & Y-Axis \\
\hline Hand Span & 1 & 0.15 & $0.6^{*}$ & $-0.20 \sim$ & -0.25 & 0.64 \\
MCP & 0.15 & 1 & $0.61^{*}$ & $-0.33 \sim$ & 0.29 & -0.21 \\
Grip & 0.60 & 0.61 & 1 & $-0.71 \sim$ & -0.49 & 0.14 \\
Strength & & & & &
\end{tabular}

$\sim$ Spearman's Rho

Table 4. Pearson Correlations for male participants $(n=6)$

\begin{tabular}{lcccccc}
\hline & Hand Span & MCP & Grip Strength & DCOT & X-Axis & Y-Axis \\
\hline Hand Span & 1 & $0.67^{\star}$ & $0.78^{\star *}$ & 0.20 & -0.64 & 0.28 \\
$\mathrm{MCP}^{\wedge}$ & 0.67 & 1 & $0.47^{\star}$ & 0.25 & -0.17 & 0.37 \\
Grip & 0.78 & 0.47 & 1 & 0.80 & -0.07 & 0.29 \\
Strength^ & & & & &
\end{tabular}

$\wedge_{\mathrm{n}}=5$

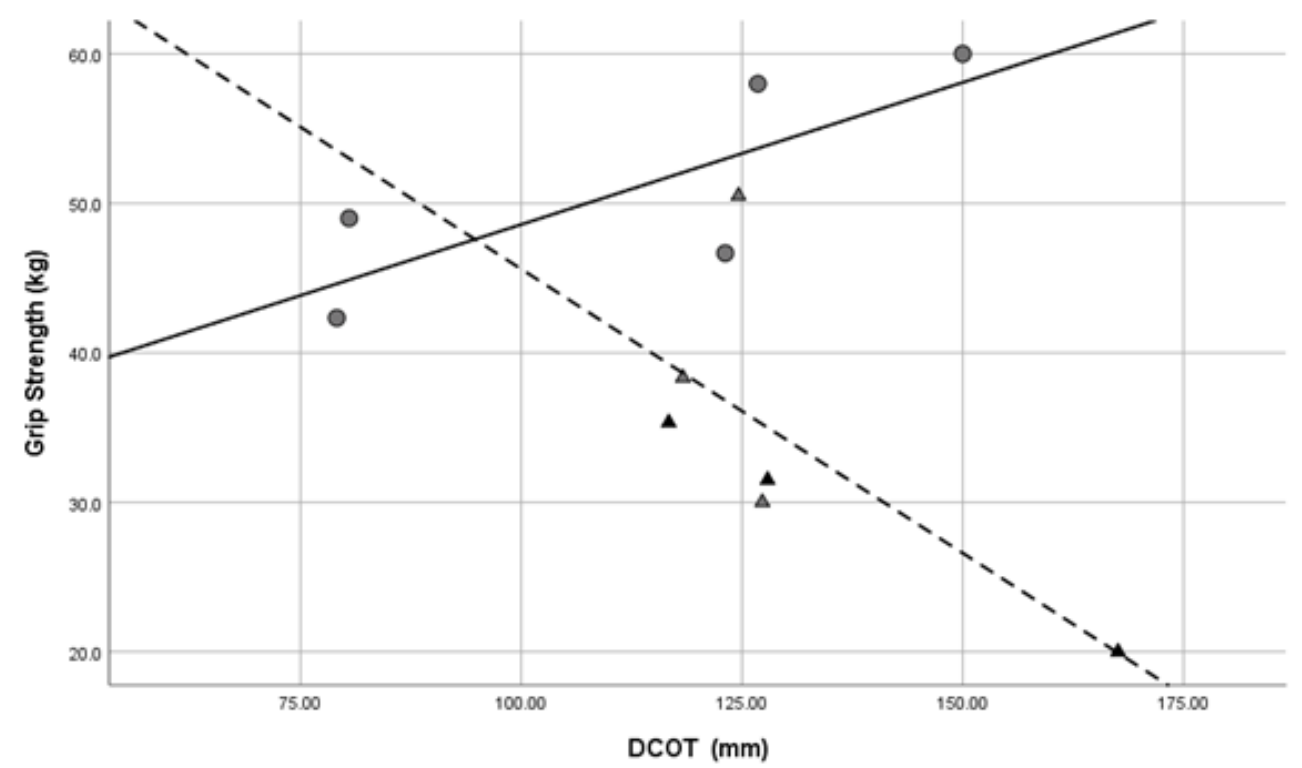

Figure 3. Trend lines of best fit for male (circles) and female (triangle) officer grip strength and DCOT 


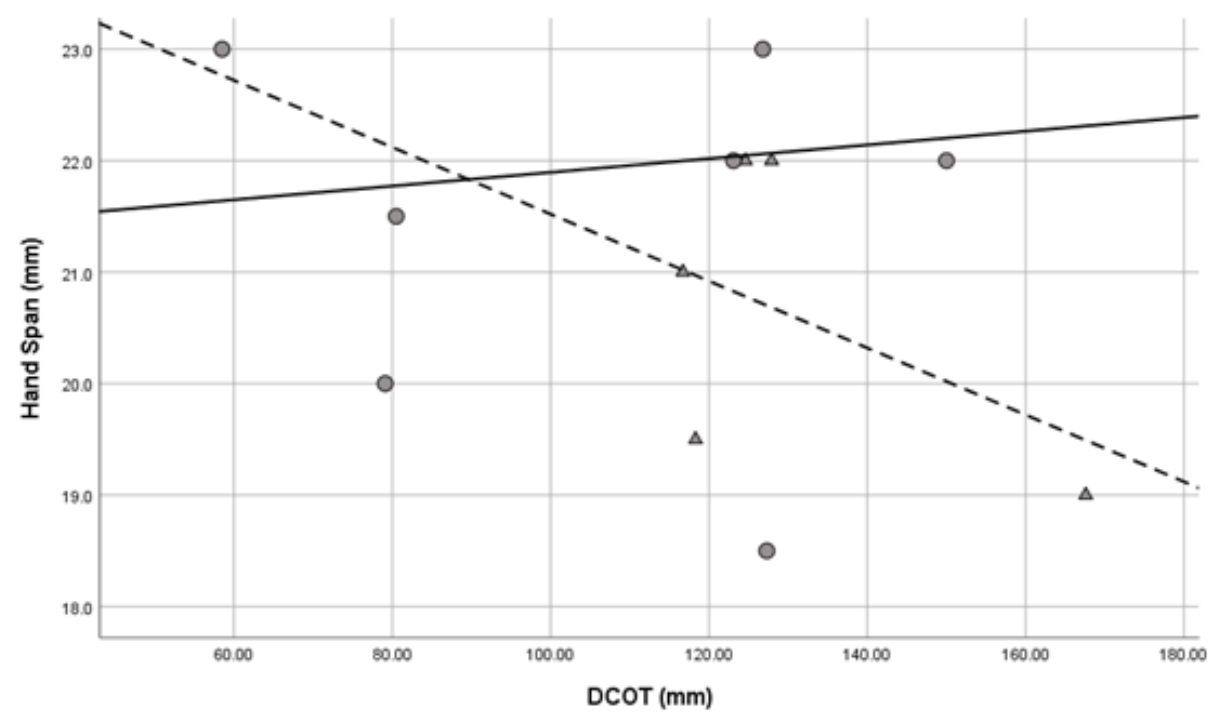

Figure 4. Trend lines of best fit for male (circles) and female (triangle) officer hand span and DCOT

\section{DISCUSSION}

The purpose of this study was to evaluate the relationships between hand size and grip strength, and pistol shooting accuracy (marksmanship) in police officers. The overall results showed that while male officers had significantly larger MCP sizes and strength there were no significant differences in hand sizes or marksmanship measures. Furthermore, these relationships (MCP sizes and strengths) no longer appeared when each sex was analysed separately. As expected, there were significant correlations between hand size (hand span and MCP) and grip strength, although again these were not related when each sex was considered separately. Finally, visual representation of each charted participant suggested a pattern, whereby as grip strength increased female officer marksmanship performance improved, while male officer performance got worse. The same trend, as could be expected given the cohort correlations between grip strength and hand span, was seen in hand span in relation to sex and marksmanship performance.

Noting the different methodology for conducting the grip strength assessment in this study, the grip strength of officers in this study was similar to those reported in other literature on police officer grip strengths (Charles \& Copay, 2001; Dawes et al., 2017; Orr et al., 2017). Furthermore, the grip strength of this smaller sample is similar to a previous study in the same population of 169 participants (cohort grip strength $=42.15 \pm 8.29 \mathrm{~kg}$ ) (Orr et al., 2017). Given the MCP measure in this study was slightly distal to that reported by Anderson and Plecas (2000) (who measured hand width across the base of the $2^{\text {nd }}$ to $5^{\text {th }}$ metacarpals), the results reported in this study are similar. Unfortunately, no other known studies reported on hand span. On this basis, the hand size and grip strength captured by this sample is akin to those found in other studies. 
However, the results of this study demonstrate interesting relationships with previous research. In contrast to the study by Orr et al., (2017), who found significant correlations between grip strength and marksmanship, this study did not. However, in this study officers fired 10 rounds at a given target. In the study by Orr et al. (2017), recruits fired 30 rounds with a dedicated scoring system of zero to four points depending on strike zone. As such, and as noted by Muirhead et al. (2019), the difference in marksmanship assessments may have contributed to these differences. Of note, however, an interesting trend was found for male officers that warrants further investigation. In male officers, as grip strength increased, marksmanship performance decreased and conversely in the female officers as grip strength increased so did marksmanship performance. When considering this relationship trend across the cohort and noting that the female officers tended towards lower grip strength scores (5/6 below the mean), the emerging $\mathrm{V}$-shape curve of the results suggests that both insufficient and excessive grip strength could have a negative impact on marksmanship performance when firing the Glock 17. A similar trend was found for hand size. This is not however, unexpected given the strong relationship between hand size and grip strength (Liao, 2016). Considering this, the standard hand grip length of the Glock 17 does not accommodate different hand sizes (and related grip strength). Therefore, a single standardised hand grip length, but not allowing for differences in the hand sizes and hand grip strengths of individual officers, may impact on officer marksmanship capability.

As was expected, and similar to other marksmanship studies (Anderson \& Plecas, 2000; Charles \& Copay, 2001; Copay \& Charles, 2001), male officers had significantly greater grip strength scores than female officers. However, while hand MCP width was significantly smaller, hand span was similar. This may be due to the anthropometrics of the sample whereby there was no significant difference in height between male and female officers. It should be noted, however, that there was a trend towards significant differences in height and hand span $(\mathrm{p}=0.0059$ and $\mathrm{p}=$ 0.068 respectively). The significant difference in grip strength and trending, but lack of, significant difference in hand span size may explain the marksmanship results of the male and female officers. Although male officers did generally have better marksmanship scores $($ mean $=103.00 \pm 35.32)$ than female officers $(130.40 \pm 18.80)$ these differences failed to reach significance $(\mathrm{p}=0.134)$.

The results of this study support the work of Copay and Charles (2001). In their study, as in this study, female officers, in general, had lower grip strength and marksmanship scores than male officers. However, when sex was considered in the regression analysis, it was found not to be a predictive factor. These data suggest that while females may have lower grip strength and marksmanship scores, grip strength, rather than sex, may be the deciding factor. Furthermore, again as suggested by Copay and Charles (2001) and emerging in this study, there may be point whereby grip strength is no longer positively associated with improved marksmanship performance and may conversely become a limiting factor. 


\section{Limitations of the study}

There are several limitations for this research notable the size of the sample group. The small sample of officers, while potentially limiting the strength of findings, did present with grip strength results similar to larger cohort sizes (Charles \& Copay, 2001; Copay \& Charles, 2001). However, studies with much larger cohorts are needed to determine if indeed a V-shaped curve does exist between grip strength, hand span and marksmanship performance.

\section{CONCLUSIONS}

Neither hand size nor grip strength had a significant impact on marksmanship even though there were strong and significant relationships between hand size (span and $\mathrm{MCP}$ ) and grip strength. Male officers with significantly greater grip strength tended to shoot better than female officers up to the point where, as their grip strength increased their shooting performance tended to decrease. For the female officers, as grip strength increased their shooting performance tended to improve. These results suggest a V-shaped curve between grip strength and marksmanship, with a potential influencing factor being the standard sizing of the pistol grip.

\section{ACKNOWLEDGMENTS}

Authors have no conflicts of interest to declare.

\section{REFERENCES}

Anderson, G. S., \& Plecas, D. B. (2000). Predicting shooting scores from physical performance data. Policing: An International Journal of Police Strategies \& Management, 23(4), 525-537. https://doi.org/10.1108/13639510010355611

Binder, A., \& Fridell, L. (1984). Lethal force as a police response. Criminal Justice Abstracts, 16(2), 250-280.

Carbone, P., Carlton, S., Stierli, M., \& Orr, R. (2014). The impact of load carriage on the marksmanship of the tactical police officer: A pilot study. Journal of Australian Strength and Conditioning, 22(2), 50-57.

Charles, M. T., \& Copay, A. G. (2001). Marksmanship skills of female police recruits: Impact of basic firearms training. International Journal of Police Science \& Management, 3(4), 303-308.

Charles, M. T., \& Copay, A. G. (2003). Acquisition of marksmanship and gun handling skills through basic law enforcement training in an American police department. International Journal of Police Science \& Management, 5(1), 16-30. https://doi. org/10.1350/ijps.5.1.16.11245 
Cohen, J. (1988). Statistical power analysis for the behavioral sciences (2nd ed.). Lawrence Earlbaum Associates.

Copay, A. G., \& Charles, M. T. (2001). The influence of grip strength on handgun marksmanship in basic law enforcement training. Policing: An International Journal of Police Strategies \& Management, 24(1), 32-39.

Dawes, J., Lockie, R. G., Kukić, F., Cvorovic, A., Kornhauser, C., Holmes, R., \& Orr, R. M. (2019). Accuracy of self-reported height, body mass and derived body mass index in a group of law enforcement officers. NBP. Nauka, bezbednost, policija, 24(2), 7-15. https://doi.org/10.5937/nabepo24-21191

Dawes, J., Orr, R., Flores, R., Lockie, R., Kornhauser, C., \& Holmes, R. (2017). A physical fitness profile of state highway patrol officers by gender and age. Annals of Occupational and Environmental Medicine, 29(16), 1-11. https://doi.org/10.1186/ s40557-017-0173-0

Dopsaj, M., Marković, S., \& Prebeg, G. (2020). New possible multidimensional models for classification of the basic level of pistol shooting skill. NBP. Nauka, bezbednost, policija, 25(2), 29-38. https://doi.org/10.5937/nabepo25-25070

Dopsaj, M., Marković, S., Umek, A., Prebeg, G., \& Kos, A. (2019). Mathematical model of short distance pistol shooting performance in experienced shooters of both gender. NBP. Nauka, bezbednost, policija, 24(3), 3-13. https://doi.org/10.5937/ nabepo24-23287

Dortkamph, M. (1987). The fitness evaluation handbook. Exact Publishing.

Geller, W. A., \& Scott, M. (1992). Deadly force: What we know: A practitioner's desk reference on police-involved shootings. Police Executive Res Forum.

Hopkins, W. (2002). A scale of magnitude for effect statistics. Retrieved October 6 from www.sportsci.org/resource/stats/index.html

Hopkins, W. G. (2004). How to interpret changes in an athletic performance test. Sport Science, 8, 1-7.

Liao, K.-H. (2016). Optimal handle grip span for maximum hand grip strength and accurate grip control strength exertion according to individual hand size. Journal of Osteoporosis and Physical Activity, 4(2), 1-6. https://doi.org/10.4172/23299509.1000178

Mathiowetz, V. (2002). Comparison of Rolyan and Jamar dynamometers for measuring grip strength. Occupational therapy international, 9(3), 201-209. https://doi. org/10.1002/oti.165

Matulia, K. J. (1985). A balance of forces: Model deadly force policy and procedure. International Association of Chiefs of Police.

Muirhead, H., Orr, R., Schram, B., Kornhauser, C., Holmes, R., \& Dawes, J. J. (2019). The relationship between fitness and marksmanship in Police Officers. Safety, 5(3), 54. http://dx.doi.org/10.3390/safety5030054 
Orr, R., Pope, R., Siterli, M., \& Hinton, B. (2017). Grip strength and its relationship to police recruit task performance and injury risk: A retrospective cohort study. International Journal of Environmental Research and Public Health, 14(8), 941. https:// doi.org/10.3390/ijerph14080941

Peolsson, A., Hedlund, R., \& Öberg, B. (2001). Intra-and inter-tester reliability and reference values for hand strength. Journal of rehabilitation medicine, 33(1), 36-41. https://doi.org/10.1080/165019701300006524 